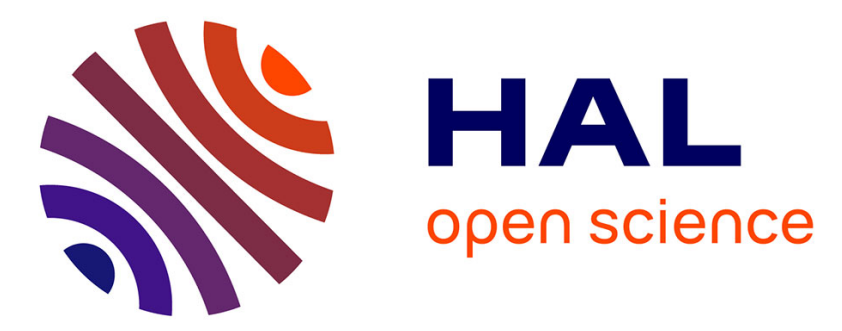

\title{
Velocimetric third-harmonic generation microscopy: micrometer-scale quantification of morphogenetic movements in unstained embryos
}

Delphine Débarre, Willy Supatto, Emmanuel Farge, Bruno B. Moulia, Marie-Claire Schanne-Klein, Emmanuel Beaurepaire

\section{To cite this version:}

Delphine Débarre, Willy Supatto, Emmanuel Farge, Bruno B. Moulia, Marie-Claire SchanneKlein, et al.. Velocimetric third-harmonic generation microscopy: micrometer-scale quantification of morphogenetic movements in unstained embryos. Optics Letters, 2004, 29, pp.2881-2883. 10.1364/OL.29.002881 . hal-00681942

\section{HAL Id: hal-00681942 \\ https://hal.science/hal-00681942}

Submitted on 28 Oct 2013

HAL is a multi-disciplinary open access archive for the deposit and dissemination of scientific research documents, whether they are published or not. The documents may come from teaching and research institutions in France or abroad, or from public or private research centers.
L'archive ouverte pluridisciplinaire HAL, est destinée au dépôt et à la diffusion de documents scientifiques de niveau recherche, publiés ou non, émanant des établissements d'enseignement et de recherche français ou étrangers, des laboratoires publics ou privés. 


\title{
Velocimetric third-harmonic generation microscopy: micrometer-scale quantification of morphogenetic movements in unstained embryos
}

\author{
Delphine Débarre \\ Laboratory for Optics and Biosciences, Centre National de la Recherche Scientifique, Institut National de la Santé et de la Recherche \\ Médicale, Ecole Polytechnique, F-91128 Palaiseau, France
}

Willy Supatto and Emmanuel Farge

Mechanics and Genetics of Developmental Embryology, Centre National de la Recherche Scientifique, Curie Institute, 11 Rue Pierre et Marie Curie, F-75005 Paris, France

Bruno Moulia

Biomechanics Group, Institut National de la Recherche Agronomique, 234 Avenue du Brézet, F-63039 Clermont-Ferrand, France

\section{Marie-Claire Schanne-Klein and Emmanuel Beaurepaire}

Laboratory for Optics and Biosciences, Centre National de la Recherche Scientifique, Institut National de la Santé et de la Recherche Médicale, Ecole Polytechnique, F-91128 Palaiseau, France

Received June 30, 2004

\begin{abstract}
We demonstrate the association of third-harmonic generation (THG) microscopy and particle image velocimetry (PIV) analysis as a novel functional imaging technique for automated micrometer-scale characterization of morphogenetic movements in developing embryos. Using a combined two-photon-excited fluorescence and THG microscope, we characterize the optical properties of Drosophila embryos and show that sustained THG imaging does not perturb sensitive developmental dynamics. Velocimetric THG imaging provides a quantitative description of the dynamics of internal structures in unstained wild-type and mutant embryos. (C) 2004 Optical Society of America

OCIS codes: $180.0180,190.4160,190.4180,180.6900,170.7050,170.1420$.
\end{abstract}

The development of animal embryos exhibits a complex ensemble of cell movements that are highly regulated in time and space. Investigating these complex dynamic processes remains a challenge in biology. ${ }^{1}$ In particular, control of morphogenetic movements involved in Drosophila melanogaster embryo development serves as a major model for developmental genetics. ${ }^{2}$ However, Drosophila embryos exhibit fast development dynamics and are highly scattering for visible light at early stages, which limits direct imaging of dynamic processes. In addition, fluorescent labeling can introduce unwanted perturbations and might be difficult to obtain in complex mutants. As a consequence, the mechanical behavior of specific cells and tissues is not completely understood in many cases. In this context, novel noninvasive techniques for visualizing and quantifying morphogenetic movements in vivo should have a significant effect on developmental biology.

Third-harmonic generation (THG) microscopy ${ }^{3}$ has been proposed as a novel general-purpose technique for obtaining structural images of transparent objects with micrometer three-dimensional resolution comparable with that of two-photon-excited fluorescence (2PEF) microscopy and was demonstrated recently in biological samples such as cells ${ }^{4}$ and zebra fish embryos. ${ }^{5}$ In this Letter we extend THG microscopy to the quantitative measurement of tissue velocity fields inside unstained opaque embryos by combining THG microscopy and particle image velocimetry (PIV) analysis techniques adapted from hydrodynamics.
First we use a combined 2PEF-THG approach to characterize the optical properties of Drosophila early embryos. Then we demonstrate that sustained THG embryo imaging does not perturb the dynamics of sensitive developmental processes. Finally, we illustrate velocimetric THG imaging by micrometer-scale quantification of morphogenetic movements in live unstained wild-type and mutant embryos.

We characterized early Drosophila embryo properties (Fig. 1) by using a custom-built combined 2PEF-THG microscope incorporating a femtosecond titanium:sapphire oscillator (Coherent, Inc.), an optical parametric oscillator (APE), galvanometer mirrors (GSI Lumonics), water-immersion objectives (Olympus), and photon-counting photomultiplier modules (Electron Tubes). 2PEF was epidetected when green fluorescent protein- (GFP-) expressing embryos were excited at $820-920 \mathrm{~nm}$. Alternatively, THG was detected in the transmitted direction when embryos were excited at $1180 \mathrm{~nm}$. In either case, the signal was selected by use of appropriate filters (Chroma).

Combined 2PEF-THG imaging of embryos with nuclear GFP labeling illustrates the complementarity of these imaging modalities (Fig. 1). At the onset of gastrulation (the universal primary morphogenetic process in animal development), Drosophila embryos comprise a single cell layer surrounding a heterogeneous medium (yolk), which is totally opaque in transmitted light microscopy. As expected, GFPlabeled nuclei produce a strong $2 \mathrm{PEF}$ signal when they are excited at $820 \mathrm{~nm}$. THG is enhanced for 


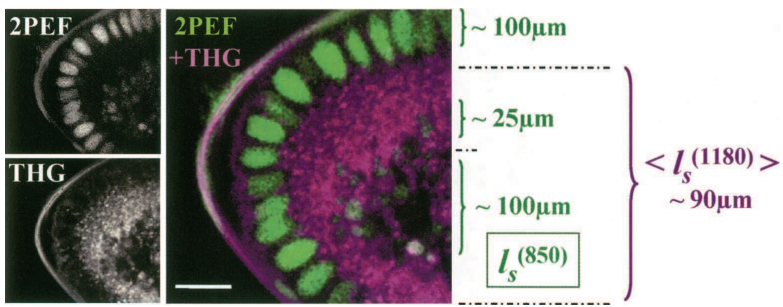

Fig. 1. Optical properties of early Drosophila embryos investigated by $2 \mathrm{PEF}-\mathrm{THG}$ microscopy. 2PEF imaging (820-nm excitation) reveals GFP-labeled nuclei and endogenous yolk fluorescence. Lipid droplets are a major source of contrast in THG images (1180-nm excitation). $l_{s}$, scattering mean free path (see text); scale bar, $15 \mu \mathrm{m}$.

micrometer-sized optical heterogeneities. ${ }^{6}$ When embryos are illuminated at $1180 \mathrm{~nm}$, lipid droplets that are present around the nuclei and at the periphery of the central yolk region produce a readily detectable THG signal $\left(5-10 \times 10^{6}\right.$ detected photons/s in our imaging conditions). These droplets have typical sizes of the order of $0.5 \mu \mathrm{m},{ }^{7}$ which contribute to THG signal enhancement. ${ }^{6}$

The key parameter for nonlinear microscopy of tissues is the scattering mean free path of excitation light $l_{s}{ }^{\text {(ex) }}$, because it defines the increase in laser power that is necessary to compensate for the scattering of excitation light with depth. ${ }^{8}$ When absorption is neglected and with the assumption of constant collection efficiency, the detected signal for an $n$ th-order process locally scales as $\exp \left[-z / l_{s}{ }^{(\mathrm{ex})}\right]^{n}$ for a given incident power, where $z$ is the imaging depth. We estimated $l_{s}{ }^{(850)}$ (850-nm excitation) at different depths within embryos at the onset of gastrulation and found that three regions with distinct optical properties could be defined: (i) the peripheral region (nuclei) for which $l_{s}{ }^{(850)} \sim 100 \mu \mathrm{m}$, (ii) a highly scattering region at the yolk surface $\left[l_{s}{ }^{(850)} \sim 25 \mu \mathrm{m}\right]$, and (iii) the internal yolk $\left[l_{s}{ }^{(850)} \sim 100 \mu \mathrm{m}\right]$. As is evident from THG images, the highly scattering nature of the yolk's surface is related to the density of lipid droplets in this region, which strongly hampers inner tissue imaging by conventional techniques. We estimated an average scattering length $\left\langle l_{s}{ }^{(1180)}\right\rangle$ for THG imaging over the entire yolk region (see Fig. 1). We found that $\left\langle l_{s}{ }^{(1180)}\right\rangle \sim$ $90 \mu \mathrm{m}$, indicating that THG microscopy performs well deep within embryos owing to the reduced scattering of excitation wavelengths in the 1100-1300-nm range.

Rapid THG imaging of embryos with $\sim 80-\mathrm{MHz}$ pulse trains requires instantaneous intensities in the $\mathrm{TW} / \mathrm{cm}^{2}$ regime $(90-\mathrm{mW}$ average power with a $0.8-\mathrm{N}$.A. objective). Although THG microscopy is believed to be noninvasive because harmonic generation processes do not involve energy deposition in the sample, the possibility of laser-induced perturbation with such intensities cannot be excluded without experimental evidence. As a matter of fact, we observed bubble formation in isolated cells with slightly higher pulse energy in the same wavelength range (data not shown). To establish the validity of THG microscopy for measuring the dynamics of developmental processes we monitored the process of cellularization, a critical and temperature-sensitive dynamic event of embryonic cells. Cellularization involves oocyte plasma membrane folding between nuclei, resulting in the formation of individual cells. The rate and the completion of cellularization front invagination (CFI) are sensitive indicators of the integrity of cytoskeleton dynamics. ${ }^{9}$ We found that CFI can be followed in vivo by THG microscopy because it induces changes in the distribution of lipid droplets about the nuclei. We compared the CFI rate in control cytoskeleton-labeled GFP (sGMCA) embryos by using $2 \mathrm{PEF}$ microscopy and in unlabeled wild-type embryos by THG microscopy. As illustrated in Fig. 2, we found similar dynamics and phase durations for the two imaging modalities. These data also corroborate transmitted-light measurements (not shown). We conclude that for these imaging conditions $(1180 \mathrm{~nm}$, $80 \mathrm{MHz}, 0.9 \mathrm{~nJ}, 0.8$ N.A., $120-\mu \mathrm{m} / \mathrm{ms}$ scan speed, $20 \mathrm{~s}$ between successive images), sustained THG imaging does not induce significant perturbation. Moreover, we observed a normal survival rate $(85 \pm 3 \%)$ after long-term imaging, which further confirms the validity of THG microscopy for measuring embryo developmental dynamics.

To provide a micrometer-scale quantitative description of morphogenetic movements, we estimated instantaneous velocity fields from THG image sequences of developing embryos by use of PIV, ${ }^{10}$ a technique commonly used in hydrodynamics. PIV relies on correlation calculations and can extract velocimetric data with subpixel resolution even when moving structures are not clearly defined in the images, in contrast to standard tracking methods. THG microscopy is sensitive to micrometer-sized optical inhomogeneities and provides rich structural information throughout entire unstained embryos. As a consequence, THG image sequences are ideally suited for correlation analysis (Fig. 3). PIV analysis was performed with the MatPIV software package ${ }^{11}$ with $64 \times 64$ pixel interrogation windows. ${ }^{10}$ As shown in Fig. 3, velocimetric analysis of THG data provides information about tissue

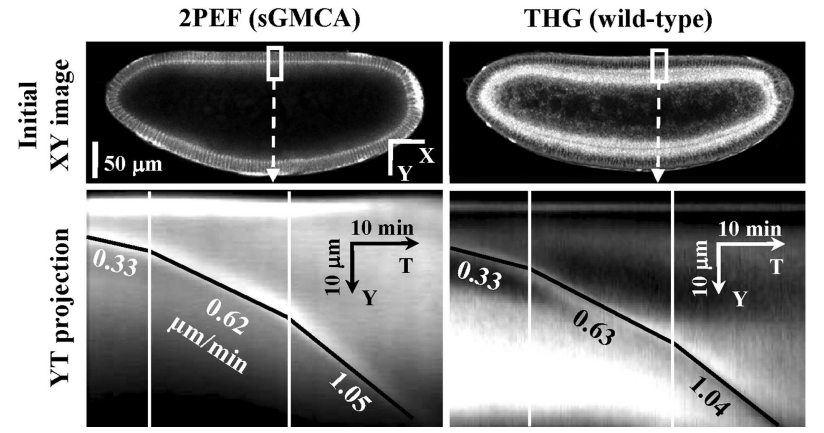

Fig. 2. Cellularization dynamics were followed at $19{ }^{\circ} \mathrm{C}$ in control sGMCA embryos by $2 \mathrm{PEF}$ microscopy (920-nm excitation) and in wild-type embryos by THG microscopy (1180-nm excitation). Image sequences were recorded, and space-time (YT) projections of the signal in the white rectangle areas were extracted. In the YT representation, slopes reflect the rate of CFI; see text. Similar speeds (indicated in micrometers per minute) are found in both experiments, which show that sustained THG imaging does not perturb the dynamics of this sensitive, temperaturedependent process. 


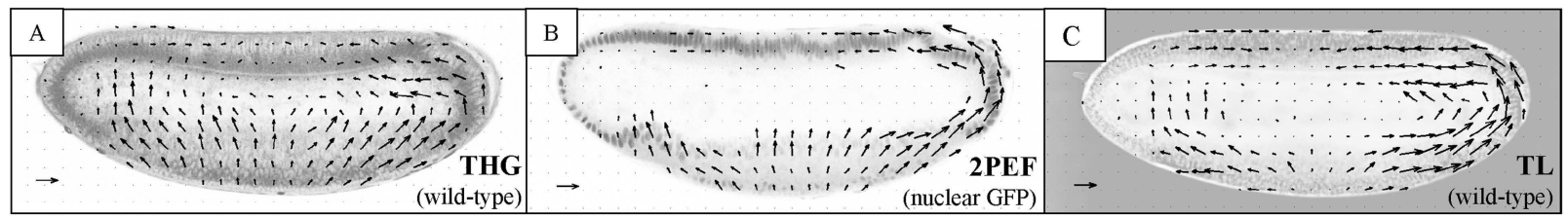

Fig. 3. A, Velocimetric THG microscopy provides in vivo a micrometer-scale description of morphogenetic movements through entire unstained wild-type embryos. B, Velocimetric analysis of 2PEF images of a nuclei-labeled embryo. C, Velocimetric analysis of transmitted-light (TL) images of an unstained embryo does not provide three-dimensional sectioning. Scale arrow, $5 \mu \mathrm{m} / \mathrm{min}$. One image was recorded every $20 \mathrm{~s}$, with $0.6-\mu \mathrm{m}$ pixel size.

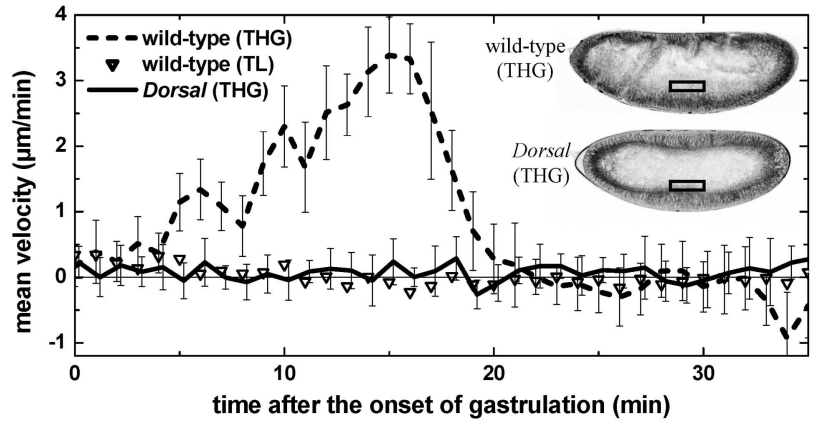

Fig. 4. THG microscopy provides micrometer-scale quantification of disrupted morphogenetic movements in unstained mutant embryos. The mean velocity in the area defined by a black rectangle, as determined by velocimetric THG in a wild-type embryo, is shown as a function of time. The same measurements in a Dorsal embryo with disrupted movements and in a wild-type embryo from transmitted-light (TL) microscopy are also shown. Velocities are projected in the vertical direction, positive up.

movements similar to that obtained from GFP lines without the need to stain structures selectively. It has the additional benefit of providing deeper imaging in unstained areas. Despite the highly scattering nature of early Drosophila embryos, clear velocimetric THG whole-embryo images could be obtained down to the equator within rapid acquisition times ( $2.3 \mathrm{~s}$; Fig. 3A), providing information that is not visible in $2 \mathrm{PEF}$ and transmitted-light images. Since morphogenetic movements are symmetric relative to the equatorial plane (antero-posterior, dorso-ventral axis), they all occur within the accessible volume of view. As is apparent from Fig. 3, velocimetric THG imaging provides a simultaneous description of tissue and internal structure dynamics and reveals their coupling.

We expect that a major application of this technique will be micrometer-scale characterization of morphogenetic movements in mutant embryos, for which GFP constructs can be difficult to obtain. We illustrate this point by comparing developmental dynamics of unstained wild-type embryos and unstained embryos from dorsal mutant mothers (so-called dorsal embryos). ${ }^{2} \quad$ Dorsal embryos lack dorso-ventral polarization and exhibit disrupted movements. Figure 4 illustrates that velocimetric THG imaging clearly extracts distinct velocity fields from internal regions of wild-type and dorsal embryos during ventral furrow invagination and that this information is not accessible to conventional imaging techniques.

Our experiments establish the combination of THG microscopy and PIV as a functional imaging technique for the micrometer-scale characterization of morphogenetic movements in live unstained embryos. We showed that this approach is applicable to early Drosophila embryos, a major model in developmental genetics that is difficult to observe with conventional imaging techniques. Our combined $2 \mathrm{PEF}-\mathrm{THG}$ data provide insight about optical properties of embryos. Importantly, we showed that sustained THG imaging does not introduce significant perturbation to sensitive dynamic processes under practical imaging conditions. Thus, biologically relevant quantitative descriptions of morphogenetic movements can be obtained in both unstained wild-type and mutant embryos. This versatile approach should find a broad range of applications in developmental biology.

We thank Catherine Schaffner, Marcel Bierry, JeanMarc Sintes, and Xavier Solinas for technical assistance and Eric Brouzés and Charlotte Py for fruitful discussions. We are grateful to Daniel P. Kiehart and Ruth A. Montague for the gift of the sGMCA transgenic line. This work was supported by the Délégation Générale pour l'Armement. E. Beaurepaire's e-mail address is emmanuel.beaurepaire@polytechnique.fr.

\section{References}

1. L. Solnica-Krezel and S. Eaton, Development 130, 4229 (2003).

2. M. Bate and A. Martinez-Arias, The Development of Drosophila melanogaster (Cold Spring Harbor Laboratory Press, New York, 1993).

3. Y. Barad, H. Eisenberg, M. Horowitz, and Y. Silberberg, Appl. Phys. Lett. 70, 922 (1997).

4. D. Yelin and Y. Silberberg, Opt. Express 5, 196 (1999), http://www.opticsexpress.org.

5. S.-W. Chu, S.-Y. Chen, T.-H. Tsai, T.-M. Liu, C.-Y. Lin, H.-J. Tsai, and C.-K. Sun, Opt. Express 11, 3093 (2003), http://www.opticsexpress.org.

6. J.-X. Cheng and X. S. Xie, J. Opt. Soc. Am. B 19, 1604 (2002).

7. M. A. Welte, S. P. Gross, M. Postner, S. M. Block, and E. F. Wieschaus, Cell 92, 547 (1998).

8. M. Oheim, E. Beaurepaire, E. Chaigneau, J. Mertz, and S. Charpak, J. Neurosci. Methods 112, 205 (2001).

9. A. Royou, C. Field, J. C. Sisson, W. Sullivan, and R. Karess, Mol. Biol. Cell 15, 838 (2004).

10. M. Raffel, C. Willert, and J. Kompenhans, Particle Image Velocimetry: A Practical Guide (SpringerVerlag, Berlin, 1998).

11. J. K. Sveen, "An introduction to MatPIV v. 1.6.1," eprint series (Department of Mathematics, University of Oslo, Oslo, Norway, 2004). 